GUTPA-96-4-2

NBI-HE-96-15

\title{
Fermion Masses and Anti-grand Unification
}

\author{
C. D. Froggatt ${ }^{1}$, H. B. Nielsen ${ }^{2}$ and D. J. Smith ${ }^{2}$ \\ ${ }^{1}$ Department of Physics and Astronomy, University of Glasgow, \\ Glasgow G12 8QQ, UK \\ 2 Niels Bohr Institute, Blegdamsvej 17-21, \\ DK 2100 Copenhagen, Denmark
}

\begin{abstract}
We present an extension of the Standard Model (SM) without supersymmetry, which we use to calculate order of magnitude values for the elements of the mass matrices in the SM. In our model we can fit the 9 quark and lepton masses and 3 mixing angles using only 3 free parameters, with the overall mass scale set by the electroweak interaction. The specific model described here has the anti-grand unified gauge group $S M G^{3} \otimes U(1)_{f}$ at high energies where $S M G \equiv$ $S U(3) \otimes S U(2) \otimes U(1)$ is the SM gauge group. The SM fermions are placed in representations of the full gauge group so that they do not produce any anomalies. It is pointed out that the same results can be obtained in an anomaly free $S M G \otimes U(1)^{3}$ model.
\end{abstract}




\section{Introduction}

In the Standard Model (SM) all the fermions get a mass via the SM Higgs mechanism. The Higgs sector of the SM is not satisfactory for two reasons. First there is a different Yukawa coupling for each fermion and these couplings make up more than half of the free parameters in the SM. Second, it would seem natural that these couplings should all be similar, probably of order 1 , since there is no reason in the SM for the Higgs field to prefer to couple to one fermion rather than another. However, this is clearly not the case experimentally where we know there is a huge range of Yukawa couplings from the top quark with a Yukawa coupling of order 1 to the electron with a Yukawa coupling of order $10^{-5}$. It is this problem that we wish to address in this paper and we will introduce an extension of the SM where such a large range of Yukawa couplings is natural, due to the existence of new approximately conserved chiral gauge quantum numbers which protect the fermions from gaining a mass. In our model all the elements of the Yukawa matrices (except for one element which leads to the unsuppressed top mass) are suppressed (relative to the assumed natural order 1) by a product of the ratios of the vacuum expectation values (VEVs) of the Higgs fields required to break the extended gauge symmetry to the fundamental scale. In this way we can express very small numbers such as $10^{-5}$ as the product of 5 numbers of order $10^{-1}$. This type of approach to mass protection has been considered previously, using abelian chiral charges, for the SM [1, 2] and more recently for the minimal supersymmetric SM [3]-[6]. Here we consider the so-called anti-grand unification model [7] based on the gauge group $S M G^{3} \otimes U(1)_{f}$, where $S M G=S U(3) \otimes S U(2) \otimes U(1)$. In fact the same model was considered in 8 but in a more abstract manner. In this paper we further specify the model by actually making a choice of Higgs particles and we find a much better fit. Some other recent attempts to model the fermion masses and mixing angles are reviewed in [9].

\section{$2 \quad$ A Realistic Model}

A natural way to explain why there are small Yukawa couplings in the SM is to assume that the SM is just a low energy effective theory. Then the smallness of some SM Yukawa couplings can be explained by suppressing the corresponding interactions in the full theory. A simple way to do this is to extend the SM gauge group and include the SM fermions in this group so that the quantum number differences between the left-handed and right-handed fermions are not the same as they are in the SM (in the sense that we introduce new quantum numbers in the full gauge group but, of course, the SM quantum numbers which will be produced by particular combinations of these quantum numbers will be the usual SM quantum numbers for all the fermions). In this way we can still arrange that

the left-handed and right-handed top quarks should differ by just the quantum 


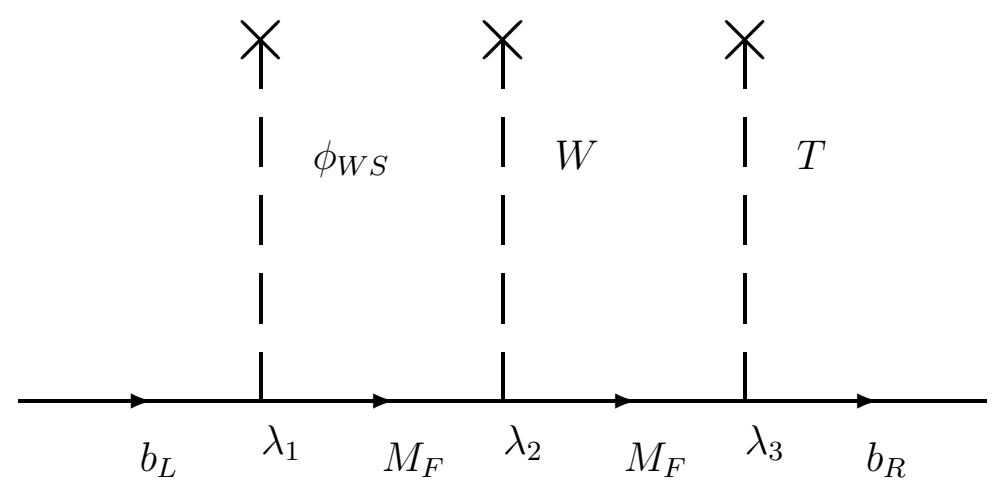

Figure 1: Feynman diagram for bottom quark mass in the full theory. The crosses indicate the couplings of the Higgs fields to the vacuum.

numbers of the Weinberg-Salam Higgs field, and so the top quark mass will not be suppressed. However, in a realistic model where all the fundamental Yukawa couplings are of order 1, no other combination of left-handed and right-handed fermions should differ by these quantum numbers, as no other fermion has the same order of magnitude mass as the top quark. As long as the new chiral gauge quantum numbers are conserved, the other fermion mass terms are forbidden. For these other fermion masses we have to introduce the new Higgs fields, which spontaneously break the extended gauge symmetry and allow the transitions giving mass terms in the SM. For example, consider the bottom quark mass. In the SM this requires a bottom quark Yukawa coupling constant significantly less than 1. However, if we consider that in the full theory the transition amplitude between the left-handed and right-handed bottom quarks is given by the Feynman diagram in fig. 1, we no longer need Yukawa couplings less than 1. If we assume that all the fundamental Yukawa coupling constants are of order unity, $\lambda_{i} \sim 1$, this diagram gives the following relation for the effective SM bottom Yukawa coupling constant:

$$
h_{b} \approx \frac{<W><T>}{M_{F}} \frac{<M_{F}}{M_{s}}
$$

where $\langle W\rangle$ and $\langle T\rangle$ are simply the VEVs of the new Higgs fields $W$ and $T$, and $M_{F}$ is the (fundamental) mass scale of the intermediate fermions. Henceforth we will consider the VEVs of all the new Higgs fields measured in units of $M_{F}$. The results for the fermion masses are rather insensitive to the precise value of $M_{F}$ but, in the spirit of the anti-grand unification model [7], we shall assume that $M_{F}$ is approximately equal to the Planck mass. This means that the above relation, eq. (11), will be taken to hold at the Planck scale.

For the smallest masses we shall require several such Higgs fields to be used rather than just one or two with a very small VEV. To calculate the effective Yukawa coupling in the SM, we simply multiply the VEVs of all the new Higgs fields needed to generate the interaction in the full theory. By using different 
combinations of a few Higgs fields we can reduce the number of free parameters. So, in a realistic model, the range of masses would be explained using a few Higgs fields with VEVs within an order of magnitude of the fundamental scale $M_{F}$.

To make such a model, we must first choose a gauge group which contains the SM gauge group (SMG). Then we must put the SM fermions in the group so that they no longer have the same quantum number differences between left and right components for different generations. Finally, we need to choose some Higgs fields to break the group down to the SMG and also give realistic masses to the fermions, assuming that all the fundamental Yukawa couplings are of order 1. We also need suitable intermediate fermions for diagrams such as fig. 1. These should be vector-like Dirac fermions with a mass of order $M_{F}$. We shall assume that all such fermions required are actually present; assuming some not to be present would cause extra suppression of some transitions.

\section{The Anti-grand Unification Model}

The anti-grand unification model has previously been considered as a candidate for explaining the fermion masses [8]. The gauge group for the model is:

$$
G=S M G_{1} \otimes S M G_{2} \otimes S M G_{3} \otimes U(1)_{f}
$$

where we have defined:

$$
S M G_{i}=S U(3)_{i} \otimes S U(2)_{i} \otimes U(1)_{i}
$$

The three $S M G_{i}$ groups will be broken down to their diagonal subgroup, which is the gauge group of the SM. The $U(1)_{f}$ group will be totally broken. This gauge group (strictly speaking without the $U(1)_{f}$ group) has been used to successfully predict the values of the running gauge coupling constants in the SM at the Planck scale[7], as critical couplings estimated using lattice gauge theory.

We put the SM fermions into this group in an obvious way. We have one generation of fermions coupling to each $S M G_{i}$ in exactly the same way as they would couple to the SMG in the SM. The broken chiral gauge quantum numbers of the quarks and leptons under the symmetry groups $S M G_{i}$ can readily explain the mass differences between fermion generations but cannot explain all the mass splittings within each generation, such as the ratio of the top and bottom quark masses. It is for this reason that the abelian flavour group $U(1)_{f}$ is introduced. We then choose $U(1)_{f}$ charges with the constraint that there should be no anomalies and no new mass-protected fermions. This leads us almost uniquely to the set of charges shown in table 1. We have labelled the fermions coupling to $S M G_{i}$ by the names of the ' $\mathrm{i}$ 'th generation of SM fermions. However, this is just a

method of labelling the representations of the full gauge group and as we will show later, for example, the fermion we have labelled $c_{R}$ will in fact turn out to be the right-handed top quark in the SM. 
Table 1: $U(1)_{f}$ charges of the fermions.

\begin{tabular}{|c|c|c|c|c|c|}
\hline Fermion & $Q_{f}$ & Fermion & $Q_{f}$ & Fermion & $Q_{f}$ \\
\hline$u_{L}$ & 0 & $c_{L}$ & 0 & $t_{L}$ & 0 \\
\hline$u_{R}$ & 0 & $c_{R}$ & 1 & $t_{R}$ & -1 \\
\hline$d_{R}$ & 0 & $s_{R}$ & -1 & $b_{R}$ & 1 \\
\hline$e_{L}$ & 0 & $\mu_{L}$ & 0 & $\tau_{L}$ & 0 \\
\hline$e_{R}$ & 0 & $\mu_{R}$ & -1 & $\tau_{R}$ & 1 \\
\hline
\end{tabular}

Now we must choose appropriate Higgs fields to break $G$ down to the SMG. The quantum numbers of the fermion fields are determined by the theoretical structure of the model (in particular the requirement of anomaly cancellation), but we do have some freedom in the choice of the quantum numbers of the Higgs fields.

\subsection{Choosing Higgs Fields}

There are obviously many different ways to break down the large group $G$ to the much smaller SMG. In order to decide how we should do this, we must consider how we are going to suppress the fermion masses in order to get a realistic model. However, we can first greatly simplify the situation by considering only the $U(1)$ charges.

To give some justification for considering only the abelian charges, we first make the observation that in the SM there is a charge quantisation rule:

$$
\frac{y}{2}+\frac{1}{2} \text { "duality" }+\frac{1}{3} \text { "triality" } \equiv 0 \quad(\bmod 1)
$$

where $y$ is the conventional weak hypercharge. If this is not an accident then we must assume that some such quantisation should be present in the full theory. The simplest way to ensure this charge quantisation rule, eq. (4), is to assume that such a rule holds for each $S M G_{i}$ separately.

The important point now is that if we assume that the matter (fermion and scalar) fields belong to singlet or fundamental representations of all non-abelian gauge groups, we can use the charge quantisation rules to determine the nonabelian representations from the $U(1)_{i}$ charges $y_{i}$. So now the four abelian charges can be used to specify the complete representation of $G$. The charges of the Higgs fields are selected by examining the abelian charge differences between 
left-handed and right-handed fermions and choosing combinations of charges to allow such transitions between them. The constraint that we must eventually recover the SMG as the diagonal subgroup of the $S M G_{i}$ groups is equivalent to the constraint that all the Higgs fields (except for the Weinberg-Salam Higgs field which of course finally breaks the SMG) should have charges $y_{i}$ satisfying:

$$
y=y_{1}+y_{2}+y_{3}=0
$$

in order that their SM weak hypercharge $y$ be zero.

\subsection{The Weinberg-Salam Higgs Field}

We wish to choose the charges of the Weinberg-Salam (WS) Higgs field so that it matches the difference in charges between the left-handed and right-handed top quarks. This will ensure that the top quark mass in the SM is not suppressed relative to the WS Higgs field VEV. However, there is a problem with this. If we choose the left-handed and right-handed top quarks to be the fermions previously labelled $t_{L}$ and $t_{R}$ then we cannot suppress the bottom and tau masses. This is because the charge differences between $t_{L}$ and $t_{R}$ are the same as between $b_{L}$ and $b_{R}$ and also between $\tau_{L}$ and $\tau_{R}$.

The only solution to this problem in our model is to choose the left-handed and right-handed top quarks to be Weyl fermions from different "proto-generations", i. e. one but not both Weyl states should couple to $S M G_{3}$. In particular, we can choose the right-handed top quark to be any of the 3 fermions we have labelled $u_{R}, c_{R}$ and $t_{R}$, since these fermions will have exactly the same quantum numbers in the SM. So we will choose the top mass to be due to the transition between the fermions we have labelled $t_{L}$ and $c_{R}$.

Now it is simple to calculate the quantum numbers of the WS Higgs field. We will use a notation to label the 4 abelian charges by:

$$
\vec{Q}=\left(\frac{y_{1}}{2}, \frac{y_{2}}{2}, \frac{y_{3}}{2}, Q_{f}\right)
$$

where $\frac{y_{i}}{2}$ are the weak hypercharges with respect to the groups $U(1)_{i}$ (normalised as in the usual SM convention) and $Q_{f}$ is the charge with respect to the group $U(1)_{f}$. So the quantum numbers of the WS Higgs field are:

$$
\vec{Q}_{W S}=\vec{Q}_{c_{R}}-\vec{Q}_{t_{L}}=\left(0, \frac{2}{3}, 0,1\right)-\left(0,0, \frac{1}{6}, 0\right)=\left(0, \frac{2}{3},-\frac{1}{6}, 1\right)
$$

This means that the WS Higgs field will in fact be coloured under both $S U(3)_{2}$ and $S U(3)_{3}$. After breaking the symmetry down to the SMG, we will be left with the usual WS Higgs field of the SM and another scalar which will be an octet of $S U(3)$ and a doublet of $S U(2)$. This should not present any phenomenological problems, provided this scalar doesn't cause symmetry breaking and doesn't have a mass less than the electroweak scale. In particular an octet of $S U(3)$ cannot lead to baryon decay. 


\subsection{Choosing the Other Higgs Fields}

We can now choose the charges of the other Higgs fields in our model, by considering the charge differences between left-handed and right-handed fermions with the inclusion of the WS Higgs. Since we have the constraint of eq. (5), the charges of these Higgs fields must be chosen to span a 3 dimensional vector space of charges represented, for example, by $\frac{y_{1}}{2}, \frac{y_{3}}{2}$ and $Q_{f}$ with $\frac{y_{2}}{2}$ being determined by eq. (5). (These charges are quantised in units of $\frac{1}{6}$ for $\frac{y_{1}}{2}$ and $\frac{y_{3}}{2}$ due to eq. (4) and the $Q_{f}$ charges are normalised to be integers for fermions as in table 1). This means that we will need at least 3 Higgs fields to break the gauge group down to the SMG. This gives us a lot of freedom, so we will choose the charges on these Higgs fields by considering phenomenological relations between fermion masses.

Since we are assuming that the fundamental Yukawa couplings are of order 1 but not exactly 1 , we can only produce order of magnitude results. So we wish to choose, for example, 2 fermions with similar masses but not order of magnitude equal masses. We can then assume that the lighter fermion is suppressed relative to the heavier fermion by 1 Higgs with a VEV given approximately by the ratio of the 2 fermion masses. For example we would say that the bottom quark and tau lepton masses were of the same order of magnitude (remembering that we take all relations at the Planck scale). However we can take the following 2 ratios of effective Yukawa couplings to be significantly different from 1:

$$
\begin{aligned}
\frac{h_{c}}{h_{b}} & \equiv<W>\approx \frac{1}{5} \\
\frac{h_{\mu}}{h_{b}} & =<T>\approx \frac{1}{13}
\end{aligned}
$$

where we have defined 2 Higgs fields, $W$ and $T$, to have appropriate VEVs to cause the charm and muon to be suppressed relative to the bottom.

First we define $\vec{b}$ to be the difference in charges between $b_{L}$ and $b_{R}$ with the inclusion of the WS Higgs field. So we have:

$$
\vec{b}=\vec{Q}_{b_{L}}-\vec{Q}_{b_{R}}-\vec{Q}_{W S}
$$

Similarly we define $\vec{c}$ and $\vec{\mu}$ to be:

$$
\begin{aligned}
\vec{c} & =\vec{Q}_{c_{L}}-\vec{Q}_{t_{R}}+\vec{Q}_{W S} \\
\vec{\mu} & =\vec{Q}_{\mu_{L}}-\vec{Q}_{\mu_{R}}-\vec{Q}_{W S}
\end{aligned}
$$

Note that $\vec{c}$ has been defined using $t_{R}$ since we have essentially swapped the righthanded charm and top quarks. Also the charges of the WS Higgs field are added rather than subtracted for up-type quarks. We observe that:

$$
\vec{b}+\vec{c}+\vec{\mu}=\overrightarrow{0}
$$


Now we can express these charges in terms of those of the Higgs fields. We can define:

$$
\vec{b}=a \vec{Q}_{W}+b \vec{Q}_{T}+\vec{Q}_{X}
$$

where we have chosen the overall sign of the charges on the Higgs fields $W$ and $T$ so that $a$ and $b$ are not negative. $\vec{Q}_{X}$ is the total charges of all other Higgs fields used to suppress the bottom mass relative to the top mass. We will assume that $\vec{Q}_{X}$ cannot be expressed as a linear combination of $\vec{Q}_{W}$ and $\vec{Q}_{T}$. Now eqs. (7) and (8) require that:

$$
\begin{aligned}
\vec{c} & = \pm(a+1) \vec{Q}_{W} \pm b \vec{Q}_{T} \pm \vec{Q}_{X} \\
\vec{\mu} & = \pm a \vec{Q}_{W} \pm(b+1) \vec{Q}_{T} \pm \vec{Q}_{X}
\end{aligned}
$$

The presence of the \pm signs is due to the fact that we can use the fields $W^{\dagger}$ and $T^{\dagger}$ as well as $W$ and $T$ (unlike in supersymmetric models [3]-[6]) in Feynman diagrams like fig. 11.

So we can rewrite eq. (12) as:

$$
\left(\begin{array}{c}
3 a+1 \\
a+1 \\
a-1 \\
-a-1
\end{array}\right) \vec{Q}_{W}+\left(\begin{array}{c}
3 b+1 \\
b-1 \\
b+1 \\
-b-1
\end{array}\right) \vec{Q}_{T}+\left(\begin{array}{c}
3 \\
1 \\
1 \\
-1
\end{array}\right) \vec{Q}_{X}=\overrightarrow{0}
$$

where the 4 coefficients for each term correspond to the 4 combinations of signs in front of the terms in eqs. (14) and (15), giving 64 cases altogether.

All possible choices of coefficient of $\vec{Q}_{X}$ are non-zero and, by assumption, $\vec{Q}_{X}$ is linearly independent of $\vec{Q}_{W}$ and $\vec{Q}_{T}$; so eq. (16) cannot hold. We must therefore conclude that there are no Higgs fields other than $W$ and $T$ used to suppress the bottom quark mass relative to the top quark mass. So we must set $\vec{Q}_{X}=\overrightarrow{0}$. We can now use the fact that $a$ and $b$ are not negative, along with the assumption that $\vec{Q}_{T}$ is not directly proportional to $\vec{Q}_{W}$, to conclude that eq. (16) requires that:

$$
a=b=1
$$

and that the combination of signs is chosen so that:

$$
\begin{aligned}
\vec{b} & =\vec{Q}_{W}+\vec{Q}_{T} \\
\vec{c} & =-2 \vec{Q}_{W}+\vec{Q}_{T} \\
\vec{\mu} & =\vec{Q}_{W}-2 \vec{Q}_{T}
\end{aligned}
$$

We note that this immediately implies the reasonably good Planck scale relation:

$$
h_{b}=<W><T>\approx \frac{1}{65}
$$


It is now a simple matter to calculate the charges of the Higgs fields $W$ and $T$. We have:

$$
\vec{Q}_{W}=\frac{1}{3}(2 \vec{b}+\vec{\mu})=\left(0,-\frac{1}{2}, \frac{1}{2},-\frac{4}{3}\right)
$$

From this we can then calculate:

$$
\vec{Q}_{T}=\vec{b}-\vec{Q}_{W}=\left(0,-\frac{1}{6}, \frac{1}{6},-\frac{2}{3}\right)
$$

We notice that the charges of $W$ and $T$ do not cover the 2 dimensional space of charges $\frac{y_{3}}{2}$ and $Q_{f}$, since only even $Q_{f}$ charges can be constructed with integer numbers of these Higgs fields. Therefore, since both $W$ and $T$ have $\frac{y_{1}}{2}=0$, we will need at least 2 more Higgs fields to fully cover the 3 dimensional charge space required to break $G$ down to the SMG. We will now choose 2 more Higgs fields which, together with $W$ and $T$, will fully cover this space.

Another parameter in the SM, which is within one order of magnitude from unity, is the mixing matrix element between the 1st and 2nd generations:

$$
V_{u s} \equiv<\xi>\approx 0.22
$$

With the mass matrix texture in our model, $V_{u s}$ is approximately given by the ratio of the mass matrix transition element from $d_{L}$ to $s_{R}$ to the transition from $s_{L}$ to $s_{R}$. This means that we must have:

$$
\vec{Q}_{\xi}=\vec{Q}_{d_{L}}-\vec{Q}_{s_{L}}=\left(\frac{1}{6}, 0,0,0\right)-\left(0, \frac{1}{6}, 0,0\right)=\left(\frac{1}{6},-\frac{1}{6}, 0,0\right)
$$

We must now choose one more Higgs field to fully span the 3 dimensional space of charges. We shall choose this Higgs field, $\chi$, so that the transition from $d_{L}$ to $s_{R}$ is of the same order of magnitude as the transition from $s_{L}$ to $d_{R}$. This will lead to 2 different but comparable mechanisms for the down quark mass. So we have:

$$
<\chi>=1
$$

and the charges of $\chi$ are given by:

$$
\begin{aligned}
\vec{Q}_{\chi} & =\left[\vec{Q}_{s_{L}}-\vec{Q}_{d_{R}}\right]-\left[\vec{Q}_{d_{L}}-\vec{Q}_{s_{R}}\right] \\
& =\left[\left(0, \frac{1}{6}, 0,0\right)-\left(-\frac{1}{3}, 0,0,0\right)\right]-\left[\left(\frac{1}{6}, 0,0,0\right)-\left(0,-\frac{1}{3}, 0,-1\right)\right] \\
& =\left(\frac{1}{6},-\frac{1}{6}, 0,-1\right)
\end{aligned}
$$

We can now calculate the suppression of all elements in the Yukawa matrices. However, we must first note that, since we have used 4 Higgs fields, we cannot uniquely resolve the charge differences between left-handed and right-handed fermions. There will be some combination of the 4 Higgs field charges which will 
result in vanishing charge differences. We must find the smallest combination of the 4 Higgs fields which results in a vanishing set of charges $\vec{Q}=0$. To do this we note that all fermion $U(1)_{f}$ charge differences are quantised as integers. However, the 3 Higgs fields $W, T$ and $\xi$ can only give integer $U(1)_{f}$ charge differences which are even. Therefore we must have at least two $\chi$ Higgs fields involved in the combination. Then we can find the unique combination of the other 3 Higgs fields which, together with the two $\chi$ fields, give net vanishing charge differences $\vec{Q}=0$. Thus we find that we can only resolve charges in terms of the 4 Higgs fields modulo $2 \vec{Q}_{\chi}-2 \vec{Q}_{\xi}-9 \vec{Q}_{T}+3 \vec{Q}_{W}$. Since this involves such large powers of $T$, there is usually no ambiguity in selecting the combination of Higgs fields which suppresses the transition the least.

\section{Mass Matrices}

We can now easily calculate the entries in the mass matrices, by expressing the charge differences between the left-handed and right-handed fermions in terms of the charges of the Higgs fields. We define the mass matrices by considering the mass terms in the SM to be given by:

$$
\mathcal{L}=Q_{L} M_{u} U_{R}+Q_{L} M_{d} D_{R}+L_{L} M_{l} E_{R}+\text { h.c. }
$$

The mass matrices can be expressed in terms of Yukawa matrices and the WS Higgs VEV by:

$$
M_{f}=Y_{f} \frac{<\phi_{W S}>}{\sqrt{2}}
$$

This leads to the following order of magnitude Yukawa matrices, where we have written $W$ instead of $\langle W>$ etc. (recalling that we have set $<\chi>=1$ ).

$$
\begin{aligned}
& Y_{u}=\left(\begin{array}{ccc}
W T^{2} \xi^{2} & W T^{2} \xi & W^{2} T \xi \\
W T^{2} \xi^{3} & W T^{2} & W^{2} T \\
\xi^{3} & 1 & W T
\end{array}\right) \\
& Y_{d}=\left(\begin{array}{ccc}
W T^{2} \xi^{2} & W T^{2} \xi & T^{3} \xi \\
W T^{2} \xi & W T^{2} & T^{3} \\
W^{2} T^{4} \xi & W^{2} T^{4} & W T
\end{array}\right) \\
& Y_{l}=\left(\begin{array}{ccc}
W T^{2} \xi^{2} & W T^{2} \xi^{3} & W T^{4} \xi \\
W T^{2} \xi^{5} & W T^{2} & W T^{4} \xi^{2} \\
W T^{5} \xi^{3} & W^{2} T^{4} & W T
\end{array}\right)
\end{aligned}
$$




\section{Important Features of the Mass Matrices}

One of the most important observations is that the diagonal elements in all 3 mass matrices are the same. This is simply because the $U(1)$ charge differences between the left-handed and right-handed components of a fermion are the same for all the fermions within the same proto-generation (e.g. $b_{L}$ and $b_{R}$ have the same charge difference as $\tau_{L}$ and $\tau_{R}$ ). This leads to the prediction that the fermions within each generation should have order of magnitude degenerate masses (at the fundamental scale), unless some fermion gets its mass from an off-diagonal term. So we would predict that:

$$
m_{b} \approx m_{\tau}
$$

but $m_{t}$ will be larger since the dominant term in $M_{u}$ is off-diagonal. Similarly we make the prediction:

$$
m_{s} \approx m_{\mu}
$$

For the first generation it is more complicated. At first it appears that all 3 fermions should have the same order of magnitude mass. However, in diagonalising $Y_{d}$, there are two contributions of order $W T^{2} \xi^{2}$ (in units of $\left\langle\phi_{W S}\right\rangle / \sqrt{2}$ ) to the smallest eigenvalue $m_{d}$. These come from the element $\left(Y_{d}\right)_{11}$ and the combination $\left(Y_{d}\right)_{12}\left(Y_{d}\right)_{21} /\left(Y_{d}\right)_{22}$ respectively. The down quark mass will therefore generally be larger (by approximately a factor of 2 ) and we have:

$$
m_{d} \geq m_{u} \approx m_{e}
$$

We can make approximate predictions for the values of all the fermion masses and also for the values of the mixing angles. However, it is simpler to calculate everything directly by computer. We will do this by varying the $3 \mathrm{VEVs}$ and finding the best fit to the experimental data.

\section{Results}

Now we are able to choose specific values for the $3 \mathrm{VEVs}$ and calculate the resulting masses and mixing angles. In order to find the best possible fit we must use some function which measures how good a fit is. Since we are expecting an order of magnitude fit, this function should depend only on the ratios of the fitted masses to the experimentally determined masses. The obvious choice for such a function is:

$$
\chi^{2}=\sum\left[\ln \left(\frac{m}{m_{\exp }}\right)\right]^{2}
$$

where $m$ are the fitted masses and mixing angles and $m_{\text {exp }}$ are the corresponding experimental values. The Yukawa matrices are calculated at the fundamental scale which we take to be the Planck scale. We use the first order renormalisation group equations (RGEs) for the SM to calculate the matrices at lower scales (e.g. 
see [10\|). Running masses are calculated in terms of the Yukawa couplings at 1 $\mathrm{GeV}$ using the relation:

$$
m(1 \mathrm{GeV})=\frac{h(1 \mathrm{GeV})}{\sqrt{2}}<\phi_{W S}>
$$

where the low energy VEV of the WS Higgs is:

$$
<\phi_{W S}>=246 \mathrm{GeV}
$$

The only exception is the top quark, where the experimentally measured mass is the pole mass. For quarks, the pole mass $M$ is related to the running mass $m$, to first order, by:

$$
M=m(M)\left(1+\frac{4}{3} \frac{\alpha_{S}(M)}{\pi}\right)
$$

We cannot simply use the 3 matrices given by eqs.(30)-(32) to calculate the masses and mixing angles, since only the order of magnitude of the elements is defined. This could result in accidental cancellations if we calculated the eigenvalues and eigenvectors using these values. Therefore we calculate statistically, by giving each element a random complex phase and then finding the masses and mixing angles. We repeat this several times and calculate the geometrical mean for each mass and mixing angle. In fact we also vary the magnitude of each element randomly by multiplying by a factor chosen to be the exponential of a number picked from a Gaussian distribution with mean value 0 and standard deviation 1.

We then vary the 3 free parameters to find the best fit given by the $\chi^{2}$ function. We get the lowest value of $\chi^{2}$ for the VEVs:

$$
<W>=0.158<T>=0.081<\xi>=0.099
$$

The fitted value of $\langle\xi\rangle$ is approximately a factor of two smaller than the estimate given in eq. (24). This is mainly because there are contributions to $V_{u s}$ of the same order of magnitude from both $Y_{u}$ and $Y_{d}$. The result of the fit is shown in table 22. The experimental values were obtained from [10, 11]. This fit has a value of:

$$
\chi^{2}=1.68
$$

This is equivalent to fitting 9 degrees of freedom ( 9 masses +3 mixing angles -3 Higgs VEVs) to within a factor of 1.54 of the experimental value. This is better than would have been expected from an order of magnitude fit and should be compared with $\chi^{2}=3.7$ for the fit with only 7 degrees of freedom in [8]. 
Table 2: Best fit to experimental data. All masses are running masses at $1 \mathrm{GeV}$ except the top quark mass which is the pole mass.

\begin{tabular}{|c|c|c|}
\hline & Fitted & Experimental \\
\hline$m_{u}$ & $3.8 \mathrm{MeV}$ & $4 \mathrm{MeV}$ \\
\hline$m_{d}$ & $7.4 \mathrm{MeV}$ & $9 \mathrm{MeV}$ \\
\hline$m_{e}$ & $1.0 \mathrm{MeV}$ & $0.5 \mathrm{MeV}$ \\
\hline$m_{c}$ & $0.83 \mathrm{GeV}$ & $1.4 \mathrm{GeV}$ \\
\hline$m_{s}$ & $415 \mathrm{MeV}$ & $200 \mathrm{MeV}$ \\
\hline$m_{\mu}$ & $103 \mathrm{MeV}$ & $105 \mathrm{MeV}$ \\
\hline$M_{t}$ & $187 \mathrm{GeV}$ & $180 \mathrm{GeV}$ \\
\hline$m_{b}$ & $7.6 \mathrm{GeV}$ & $6.3 \mathrm{GeV}$ \\
\hline$m_{\tau}$ & $1.32 \mathrm{GeV}$ & $1.78 \mathrm{GeV}$ \\
\hline$V_{u s}$ & 0.18 & 0.22 \\
\hline$V_{c b}$ & 0.029 & 0.041 \\
\hline$V_{u b}$ & 0.0030 & $0.002-0.005$ \\
\hline
\end{tabular}




\section{Conclusions}

We have presented a model where we can fit the 9 fermion masses and 3 measured mixing angles using just 3 free parameters, which are the VEVs of 3 fundamental Higgs fields used to break the model down to the SM. We would like to conclude by giving evidence that this fit is significant enough to support such a model. To do this we shall highlight the important features necessary for our model to work.

In some ways our main prediction is that all 3 mass matrices have the same order of magnitudes for corresponding elements on the main diagonal. This leads to the conclusion that the 3 fermion masses within each generation should be order of magnitude degenerate. However, the off-diagonal elements are not the same in the different matrices; so it happens that in the up-type matrix an offdiagonal element dominates and gives a top quark mass larger than the bottom quark or tau lepton masses. This then leads to the charm quark also getting a mass different from the strange quark and the muon. Also, to some extent, the down quark mass is larger (statistically) than the up quark and electron masses, since it can come from the usual diagonal element or order of magnitude equal off-diagonal elements. So we can naturally explain the relations given by eqs. (33)-(35) and that $m_{t} \gg m_{b}$.

This is quite different from predictions of theories such as grand unified $S U(5)$, where there should be an exact equality between down-type quarks and the charged leptons in the same generation. We only predict order of magnitude equality and, in fact, predict that the electron should have the same order of magnitude mass as the up quark rather than the down quark.

So we would claim that the evidence for this type of model is strong. However, we cannot really claim that we could only produce such results with this gauge group. For example we could consider the gauge group $S U(3) \otimes S U(2) \otimes U(1)^{4}$ where we define the $4 U(1)$ groups in the same way as in our model. This anomaly free $S M G \otimes U(1)^{3}$ model would lead to exactly the same results, since we only used the abelian charges and defined the non-abelian representations in terms of them. But we would argue that our model was aesthetically better, since the quantisation of the weak hypercharge is more natural if we have non-abelian groups $S U(3)_{i}$ and $S U(2)_{i}$ associated with the three $U(1)_{i}$. The $U(1)_{f}$ charges are less aesthetically satisfying, because the $W$ and $T$ Higgs fields have $U(1)_{f}$ charges which are quantised in units of one-third of those of the fermions.

\section{Acknowledgements}

DJS wishes to acknowledge The Royal Society for funding. HBN acknowledges funding from INTAS 93-3316, EF contract SC1 0340 (TSTS) and Cernfølgeforskning. CF acknowledges funding from INTAS 93-3316 and PPARC GR/J21231. 


\section{References}

[1] C. D. Froggatt and H. B. Nielsen, Nucl. Phys. B147 (1979) 277; B164 (1979) 144.

[2] J. Bijnens and C. Wetterich, Nucl. Phys. B283 (1987) 237.

[3] M. Leurer, Y. Nir and N. Seiberg, Nucl. Phys. B398 (1993) 319; ibid B420 (1994) 468.

[4] L. E. Ibanez and G. G. Ross, Phys. Lett. B332 (1994) 100.

[5] P. Binétruy and P. Ramond, Phys. Lett. B350 (1995); P. Binétruy, S. Lavignac and P. Ramond, preprint LPTHE-ORSAY 95/54, hep-ph/9601243.

[6] E. Dudas, S. Pokorski and C. Savoy, Phys. Lett. B356 (1995) 45.

[7] D. L. Bennett, H. B. Nielsen and I. Picek, Phys. Lett. B208 (1988) 275; D. L. Bennett and H. B. Nielsen, Int. J. Mod. Phys. A9 (1994) 5155; L. V. Laperashvili, Yad. Fiz. 57 (1994) 501

[8] C. D. Froggatt, G. Lowe and H.B. Nielsen, Nucl. Phys. B414 (1994) 579.

[9] C. D. Froggatt, to be published in Proceedings of the Fifth Hellenic School and Workshops on Elementary Particle Physics, Corfu, 1995, ed. G. Koutsoumbas and N. Tracas, preprint GUTPA/96/02/1, hep-ph/9603432.

[10] H. Arason, D.J. Castaño, B. Kesthelyi, S. Mikaelian, E.J. Piard, P. Ramond and B.D. Wright, Phys. Rev. D46 (1992) 3945

[11] Particle Data Group, Phys. Rev. D50 (1994) 1173 